INTERNATIONAL JOURNAL OF RESEARCHES IN BIOSCIENCES, AGRICULTURE AND TECHNOLOGY C VISHWASHANTI MULTIPURPOSE SOCIETY (Global Peace Multipurpose Society) R. No. MH-659/13(N) www.vmsindia.org

\title{
HERBACEOUS FLORA OF WEEDS GROWING AT ARAI HILL, PUNE Rajendra Patil ${ }^{1}$ and Minakshi Mahajan ${ }^{2}$ \\ Amolakchand Mahavidyalaya Yawatmal ${ }^{1}$ and Fergusson College, Pune-4.
}

Abstract:

Weeds are wildly gowing plants grows along the roadsides, hills, mountains, grasslands and forests. This work was conducted to study the different weed species composition growing at ARAI Hill, Pune. The dominant weeds are from the family Asteraceae, Caesalpinaceae, Amaranthaceae, Lamiaceae. The dominating species of grasses are Heteropogon contortus (L.) P. Beauv, Melanocenchris jacquemontii Jaub. \& Spach Themeda triandra and Apluda mutica L. Herbaceous flora of weeds was recorded for three years.Luxiriously growing speces are Alternanathera sessilis (L.) DC. Crotalaria hebecarpa (DC.) ,Justicia simplex D. Don.Rudd, Hyptis suaveolens (L.) ,Parthenium histerophorus L., Rungia repens (L). Nees Poit, Sopubia delphinifolia (L.) Don,Spermacocce articularis, Spermacocce stricta L.F.,Striga densiflora (Benth)., Synedrella nodiflora (L.) Gaertn, and Cassia uniflora Mill. Weeds have ecological role in the environment. They play important role in biogeochemical cycle.

Keywords: Herbaceous flora, weeds, dominating species, ARAI hill Pune.

\section{Introduction}

The weeds are the unwanted troublesome plants. They are troublesome for wanted crops, but have ecological significance. They grow in crop field, waste land, roadside, forest in or on the water bodies. Weeds when grow in the crop field, the act as a competitor for light, space, nutrients and water, but when they grow on hills, maintain prevent soil erosion. (Cocannouer, J. A. 1964). They reduce the yield. Pathogens in the form of microorganisms take support of the weeds as a host. They can complete their life cycle in adverse aconditions very effectively than the cultivated crop. Weeds support many ecosystem services. (Marshall et. al. 2003, Eraud. et. al. 2015, Requier et.al. 2015, Rollin t. al. 2016).

Weeds acts as an indicators of the presence and quality of ground water (Chikishev, 1965). The present study was conducted to know the herbaceous flora of weeds growing on ARAI hill. ARAI known as the Automotive Research Association of India. Arai Tekdi is the highest point in the city of Pune, from where the entire horizon of the city can be enjoyed. Forest department has done some plantations work on hill. There is one quarry in which aquatic weeds grow. Water gets trapped in the small $\mathrm{t}$ renches and pits in this quarry. Due to quarry, small wetland ecosystem is created, serve the purpose of bird diversity. Many bushes and trees give shelter to the birds and insects.

\section{Materials and Methods}

ARAI hill is located in Pune district, Maharashtra. The study of herbaceous weed species was carried out for three years. The frequent visit was carried out to record the important characters of weed flora and their families were recored. The digital herbarium of all the fresh plant specimens prepared. The plants are identified by using flora (Cook,
1958, Yadav and Sardesai ( ), Ingalhallikar, 2001, Naidu (2012).

\section{Result:}

Weeds have a vital role in ecosystem. They protect and restore soil that has been left exposed. Weeds growing on hill have their role in carbon sequestration. They absorbs carbon dioxide from the air and converts it into plant tissue. After completing life cycle plants dies, or shed its leaves and undergoes degradation in biogeochemical cycle. During this process part of carbon dioxide is returned to the atmosphere and part of it remains in the soil as stabilised organic matter.

\section{References}

Chikishev, A. G. (ed.) 1965. Plant indicators of soils, rocks, and subsurface waters. $210 \mathrm{pp}$. Consultants Bureau, NY.

Cocannouer, J. A. 1964. Weeds: Guardians of the Soil. 179 pp. DeVin-Adair, NY

Cook, T. (1958). Flora of the Presidency of Bombay. Botanical Survey of India, Calcutta.

Eraud, . E. Cadet, T. Powolny, S. Gaba, F. Bretagnolle and V. Bretagnolle. (2015). Weed seeds, Not Grain, Contribute to the Diet of Wintering Skylarks in? Arable Farmlands of Western France." European Journal of Wildlife Research 61: 151-161.10.1007/S 10344-0140888-y.

Ingalhalikar, S. (2001). Flowers of Sahyadri.

Marshall, E.J.P.., V. K. Brown, N.D. Boatman, P.J. W. Lutman, G. R. Squire and L. N. Ward, (2003). " The role of weeds in supporting Biological Diversity within Crop fields." Weed Research 43: 77-89.10. 1046/j. 13653180.2003.00326.X.

Naidu, V.S.G.R. 2012, Hand Book on Weed Identification Directorate of Weed Science Research, Jabalpur, India Pp 354.

Requier, F. J. F. Odoux, T. Tamic, N. Moreau, M. Henry, A. Decourtyne and V. Breatagnolle. (2015). "Honey Bee Diet in intensive Farmland habitats reveal an unexpectedly high flower 
richness and a major role of weeds." Ecological Application 25: 881-890.10.1890/14-1011.1

Rollin, O., G. Benelli, S. Benvenuti, A. Decourtye, S. D. Wratten, A. Canale, and N. Desneaux. (2016). "Weed-insect pollinator

Table No.1: Ethnobotanical survey in Pinguli area.

\begin{tabular}{|c|c|c|}
\hline $\begin{array}{l}\text { Sr. } \\
\text { No. }\end{array}$ & $\begin{array}{l}\text { Name of the plant } \\
\text { species }\end{array}$ & Family \\
\hline 1 & $\begin{array}{l}\text { Abutulon indicum (L.) } \\
\text { Sweet. }\end{array}$ & Malvaceae \\
\hline 2 & Acalypha indica L. & Euphorbiaceae \\
\hline 3 & $\begin{array}{l}\text { Acanthospermum } \\
\text { hispidum DC. }\end{array}$ & Asteraceae \\
\hline 4 & $\begin{array}{l}\text { Achyranthes aspera } \\
\text { L. }\end{array}$ & Amaranthaceae \\
\hline 5 & $\begin{array}{l}\text { Ageratum conyzoides. } \\
\text { L. }\end{array}$ & Asteraceae \\
\hline 6 & $\begin{array}{l}\text { Ageratum } \\
\text { houstonianum Mill }\end{array}$ & Asteraceae \\
\hline 7 & $\begin{array}{l}\text { Alternanathera } \\
\text { sessilis (L.) DC. }\end{array}$ & Amarathaceae \\
\hline 8 & $\begin{array}{l}\text { Alternanthera } \\
\text { triandra Lam. }\end{array}$ & Amaranthaceae \\
\hline 9 & $\begin{array}{l}\text { Amaranthus spinosus } \\
\text { L. }\end{array}$ & Amarathaceae \\
\hline 10 & $\begin{array}{l}\text { Amaranthus viridis } \\
\text { Hook. F. }\end{array}$ & Amaranthaceae \\
\hline 11 & $\begin{array}{l}\text { Antigonon leptopus } \\
\text { Hook. \& Arn. }\end{array}$ & Polygonaceae \\
\hline 12 & Bidens pumilla & Asteraceae \\
\hline 13 & Boerhaavia diffusa L. & Nyctaginaceae \\
\hline 15 & Cassia tora L. & Caesalpinaceae \\
\hline 14 & Cassia uniflora Mill. & Caesalpinaceae \\
\hline 16 & Celosia argentea L. & Amarathaceae \\
\hline 17 & $\begin{array}{l}\text { Cleome simplicifolia } \\
\text { Hook.f. \& Thomson }\end{array}$ & Capparidaceae \\
\hline 18 & $\begin{array}{l}\text { Coccinea grandis (L.) } \\
\text { Voigt }\end{array}$ & Cucurbitaceae \\
\hline 19 & $\begin{array}{l}\text { Commelina } \\
\text { benghalensis L. }\end{array}$ & Commelinaceae \\
\hline 20 & $\begin{array}{l}\text { Commelina forskaolii } \\
\text { Vahl. }\end{array}$ & Commelinaceae \\
\hline 21 & $\begin{array}{l}\text { Crotalaria hebecarpa } \\
\text { (DC.) Rudd }\end{array}$ & Fabaceae \\
\hline 22 & $\begin{array}{l}\text { Cynotis cristata } \\
\text { (Linn).D. Don. }\end{array}$ & Commelinaceae \\
\hline 23 & $\begin{array}{l}\text { Cynotis prostrata (L). } \\
\text { Blume }\end{array}$ & Commelinaceae \\
\hline 24 & Cyperus rotundus L. & Cyperaceae \\
\hline 25 & $\begin{array}{l}\text { Desmodium } \\
\text { gangeticum ( L.) DC. }\end{array}$ & Fabaceae \\
\hline 26 & $\begin{array}{l}\text { Desmodium triflorum } \\
\text { (L.) DC }\end{array}$ & Fabaceae \\
\hline 27 & $\begin{array}{l}\text { Eclipta alba (L.) } \\
\text { Hassk }\end{array}$ & Aseraceae \\
\hline 28 & $\begin{array}{l}\text { Euphorbia geniculata } \\
\text { Orteg }\end{array}$ & Euphorbiaceae \\
\hline 29 & Euphorbia hirta L. & Euphorbiaceae \\
\hline 30 & $\begin{array}{l}\text { Euphorbia } \\
\text { microphylla Heyne ex. } \\
\text { Roth. }\end{array}$ & Euphorbiaceae \\
\hline 31 & Evolvulus alsinoides & Convolvulaceae \\
\hline
\end{tabular}

Networks as Bioindicators of Ecological sustainability in Agriculture. A Review." Agrononomy for sustainable Development 36 (8): doi:1007/s 13593-015-0342-X.

\begin{tabular}{|c|c|c|}
\hline & (L.) L. & \\
\hline 32 & $\begin{array}{l}\text { Exacum } \\
\text { pedunculatum L. }\end{array}$ & Gentiaaceae \\
\hline 33 & $\begin{array}{l}\text { Exacum pumillum } \\
\text { Griseb. }\end{array}$ & Gentianaceae \\
\hline 34 & $\begin{array}{l}\text { Gomphrena } \\
\text { decumbens Jacq. }\end{array}$ & Amaranthaceae \\
\hline 37 & $\begin{array}{l}\text { Hyptis suaveolens (L.) } \\
\text { Poit }\end{array}$ & Lamiaceae \\
\hline 38 & $\begin{array}{l}\text { Indigofera cordifolia } \\
\text { Heyne. ex Roth }\end{array}$ & Fabaceae \\
\hline 39 & $\begin{array}{l}\text { Indigofera linifolia } \\
\text { (L.f.) Retz. }\end{array}$ & Fabaceae \\
\hline 40 & $\begin{array}{l}\text { Ipomea eriocarpa } \mathrm{R} \text {. } \\
\mathrm{Br} \text {. }\end{array}$ & Convulaceae \\
\hline 41 & $\begin{array}{l}\text { Ipomea parasitica } \\
\text { (Kunth) G. Don }\end{array}$ & Convulaceae \\
\hline 42 & $\begin{array}{l}\text { Ipomoea cairica (L.) } \\
\text { Sweet }\end{array}$ & Convulaceae \\
\hline 40 & $\begin{array}{l}\text { Ipomoea hederacea } \\
\text { (L.) Jacq. }\end{array}$ & Convulaceae \\
\hline 41 & $\begin{array}{l}\text { Justicia simplex D. } \\
\text { Don. }\end{array}$ & Acanthaceae \\
\hline 42 & Lagasca molis Cav & Asteraceae \\
\hline 43 & Lantana camera L. & Verbenaceae \\
\hline 44 & $\begin{array}{l}\text { Launaea nudicaulis } \\
\text { (Linn.) Hook. f. }\end{array}$ & Asteraceae \\
\hline 45 & $\begin{array}{l}\text { Lavandula bipinnata } \\
\text { (Roth) Kuntze }\end{array}$ & Asteaceae \\
\hline 46 & $\begin{array}{l}\text { Lepidogathis cristata } \\
\text { Willd. }\end{array}$ & Acanthaceae \\
\hline 47 & Leucas aspera Link & Lamiaceae \\
\hline 48 & Linum mysoorensis & Linaceae \\
\hline 49 & $\begin{array}{l}\text { Ludwigia octovalvis } \\
\text { (Jacq.) }\end{array}$ & Onagraceae \\
\hline 50 & Mimosa pudica $L$. & Mimosaceae \\
\hline 51 & $\begin{array}{l}\text { Neanotis montholonii } \\
\text { (Hook.f.) W.H.Lewis }\end{array}$ & Rubiaceae \\
\hline 52 & $\begin{array}{l}\text { Oldenlendia } \\
\text { corymbosa L. }\end{array}$ & Rubiaceae \\
\hline 53 & Oxalis corniculata L. & Oxalidaceae \\
\hline 54 & $\begin{array}{l}\text { Parthenium } \\
\text { histerophorus L. }\end{array}$ & Asteraceae \\
\hline 55 & Passiflora foetida L. & Passifloraceae \\
\hline 56 & Phyllanthus niruri L. & Euphorbiaceae \\
\hline 57 & Physalis minima L. & Solanaceae \\
\hline 58 & $\begin{array}{l}\text { Pluchea tomentosa } \\
\text { DC. in Wight, }\end{array}$ & Asteraceae \\
\hline 59 & Portulaca oleracea L. & Portulacaceae \\
\hline 60 & $\begin{array}{l}\text { Portulaca quadrifida } \\
\text { L. }\end{array}$ & Portulacaceae \\
\hline 61 & Pulicaria wightiana & Asteraceae \\
\hline 62 & $\begin{array}{l}\text { Rhamphicarpa } \\
\text { longiflora }\end{array}$ & $\begin{array}{l}\text { Scrophullariace } \\
\text { ae }\end{array}$ \\
\hline 63 & $\begin{array}{l}\text { Rostellularia } \\
\text { procumbens (L) Nees }\end{array}$ & Acanthaceae \\
\hline
\end{tabular}




\begin{tabular}{|c|c|c|}
\hline 64 & $\begin{array}{l}\text { Ruellia humilis Pohl } \\
\text { ex Nees }\end{array}$ & Acanthaceae \\
\hline 65 & $\begin{array}{l}\text { Rungia repens (L). } \\
\text { Nees }\end{array}$ & Acanthaceae \\
\hline 66 & Sesamum indicum L. & Pedaliaceae \\
\hline 67 & Sida acuta Burm. f & Malvaceae \\
\hline 68 & $\begin{array}{l}\text { Sopubia delphinifolia } \\
\text { (L.) Don }\end{array}$ & $\begin{array}{l}\text { Scrophyulariace } \\
\text { ae }\end{array}$ \\
\hline 69 & $\begin{array}{l}\text { Spermacocce } \\
\text { articularis }\end{array}$ & Rubiaceae \\
\hline 70 & $\begin{array}{l}\text { Spermacocce stricta } \\
\text { L.F. }\end{array}$ & Rubiaceae \\
\hline 71 & $\begin{array}{l}\text { Sphaeranthus } \\
\text { indicus L. }\end{array}$ & Asteraceae \\
\hline 72 & $\begin{array}{l}\text { Spilanthes acmella } \\
\text { auct. non L. }\end{array}$ & Asteraceae \\
\hline 73 & $\begin{array}{l}\text { Striga densiflora } \\
\text { (Benth). }\end{array}$ & Orobanchaceae \\
\hline 74 & $\begin{array}{l}\text { Synedrella nodiflora } \\
\text { (L.) Gaertn. }\end{array}$ & Asteraceae \\
\hline 75 & $\begin{array}{l}\text { Tephrosia purpurea } \\
\text { (L.) Pers. }\end{array}$ & Fabaceae \\
\hline 76 & $\begin{array}{l}\text { Trichodesma indicum } \\
\text { L. }\end{array}$ & Boraginaceae \\
\hline 77 & Tricolepis glabarrima & Asteraceae \\
\hline 78 & $\begin{array}{l}\text { Tridax procumbbens } \\
\text { L. }\end{array}$ & Asteraceae \\
\hline 79 & $\begin{array}{l}\text { Triumfetta } \\
\text { rhomboidea Jacq }\end{array}$ & Tiliaceae \\
\hline 80 & Urena sinuata L. & Malvaceae \\
\hline 81 & Vicoa indica L. (DC). & Asteraceae \\
\hline 82 & $\begin{array}{l}\text { Vigna radiata (L.) R. } \\
\text { Wilczek }\end{array}$ & Fabaceae \\
\hline 83 & $\begin{array}{l}\text { Xanthium } \\
\text { strumarrium L. }\end{array}$ & Asteraceae \\
\hline \multicolumn{3}{|c|}{ Grasses- } \\
\hline 84 & & Poaceae \\
\hline
\end{tabular}

\begin{tabular}{|c|c|c|}
\hline & Apluda mutica L. & \\
\hline 85 & $\begin{array}{l}\text { Anthraxon hispidus } \\
\text { (Thunb.) Makino }\end{array}$ & Poaceae \\
\hline 86 & Chloris barbata SW. & Poaceae \\
\hline 87 & $\begin{array}{l}\text { Chrysopogon fulvus } \\
\text { (Spreng.) Chiov }\end{array}$ & Poaceae \\
\hline 88 & $\begin{array}{l}\text { Cynodon dactylon (L.) } \\
\text { Pers. }\end{array}$ & Poaceae \\
\hline 99 & Dicanthium $\mathrm{sp}$ & Poaceae \\
\hline 90 & $\begin{array}{l}\text { Echinochloa colona } \\
\text { (L.) Link. }\end{array}$ & Poaceae \\
\hline 91 & $\begin{array}{l}\text { Eleusine indica (L.) } \\
\text { Gaertner. }\end{array}$ & Poaceae \\
\hline 92 & $\begin{array}{l}\text { Enteropogon } \\
\text { dolichostachyus } \\
\text { (Lagasca) Keng ex } \\
\text { Lazarides }\end{array}$ & Poaceae \\
\hline 93 & $\begin{array}{l}\text { Eragrostis bifaria } \\
\text { (Vahl) Wight }\end{array}$ & Poaceae \\
\hline 94 & $\begin{array}{l}\text { Heteropogon contortus } \\
\text { (L.) P. Beauv }\end{array}$ & Poaceae \\
\hline 95 & Heteropogon triteceus & Poaceae \\
\hline 96 & $\begin{array}{l}\text { Melanocenchris } \\
\text { jacquemontii Jaub. \& } \\
\text { Spach }\end{array}$ & Poaceae \\
\hline 97 & $\begin{array}{l}\text { Paspalum dialatatum } \\
\text { Poir. }\end{array}$ & Poaceae \\
\hline 98 & $\begin{array}{l}\text { Paspalum distichum } \\
\text { auct. nm L. }\end{array}$ & Poaceae \\
\hline 99 & $\begin{array}{l}\text { Setaria glauca (L.) P. } \\
\text { Beauv. }\end{array}$ & Poaceae \\
\hline 100 & $\begin{array}{l}\text { Sporobolus diander } \\
\text { (Retz.) P. Beauv. }\end{array}$ & Poaceae \\
\hline 101 & Themeda triandra & Poaceae \\
\hline
\end{tabular}

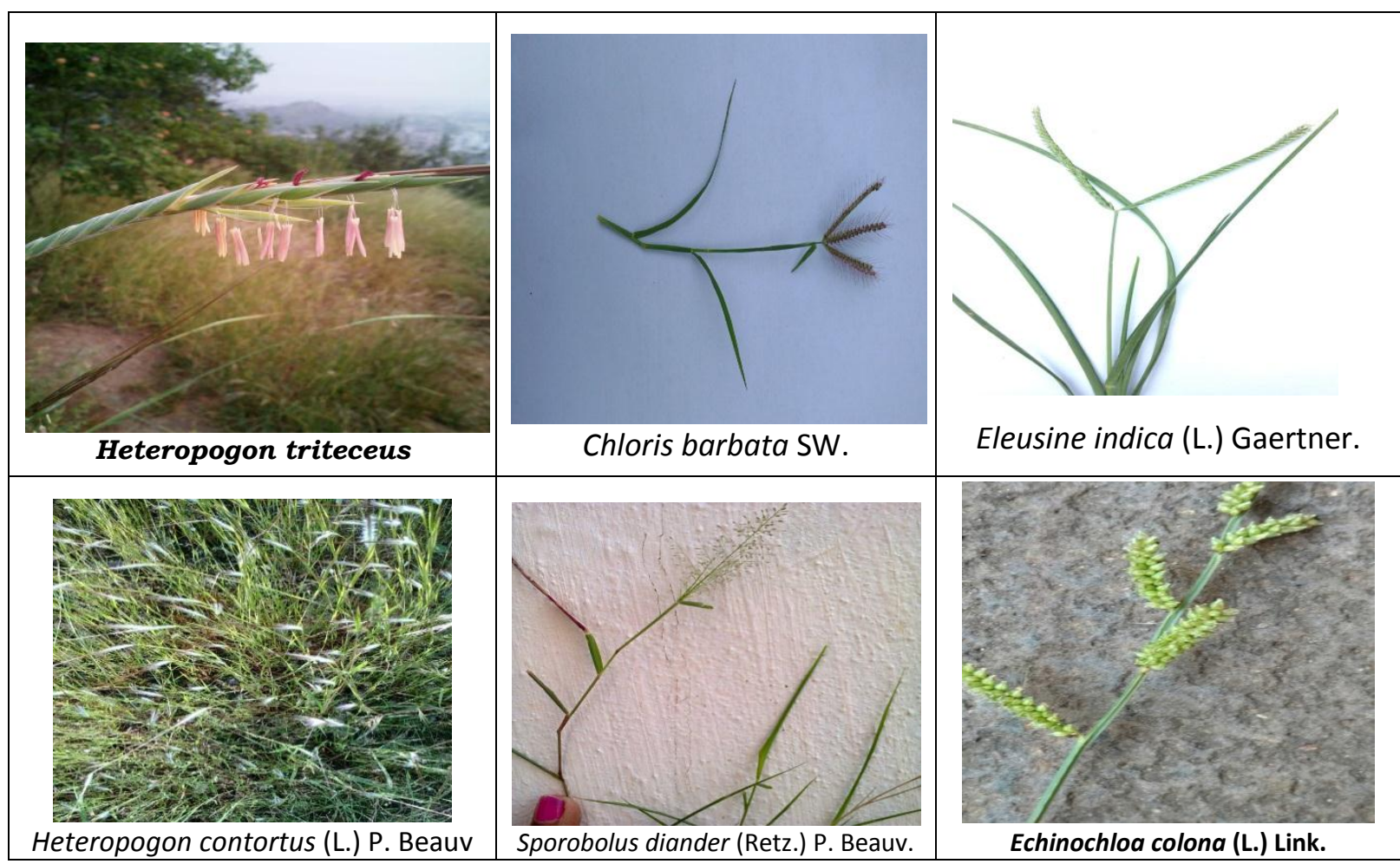




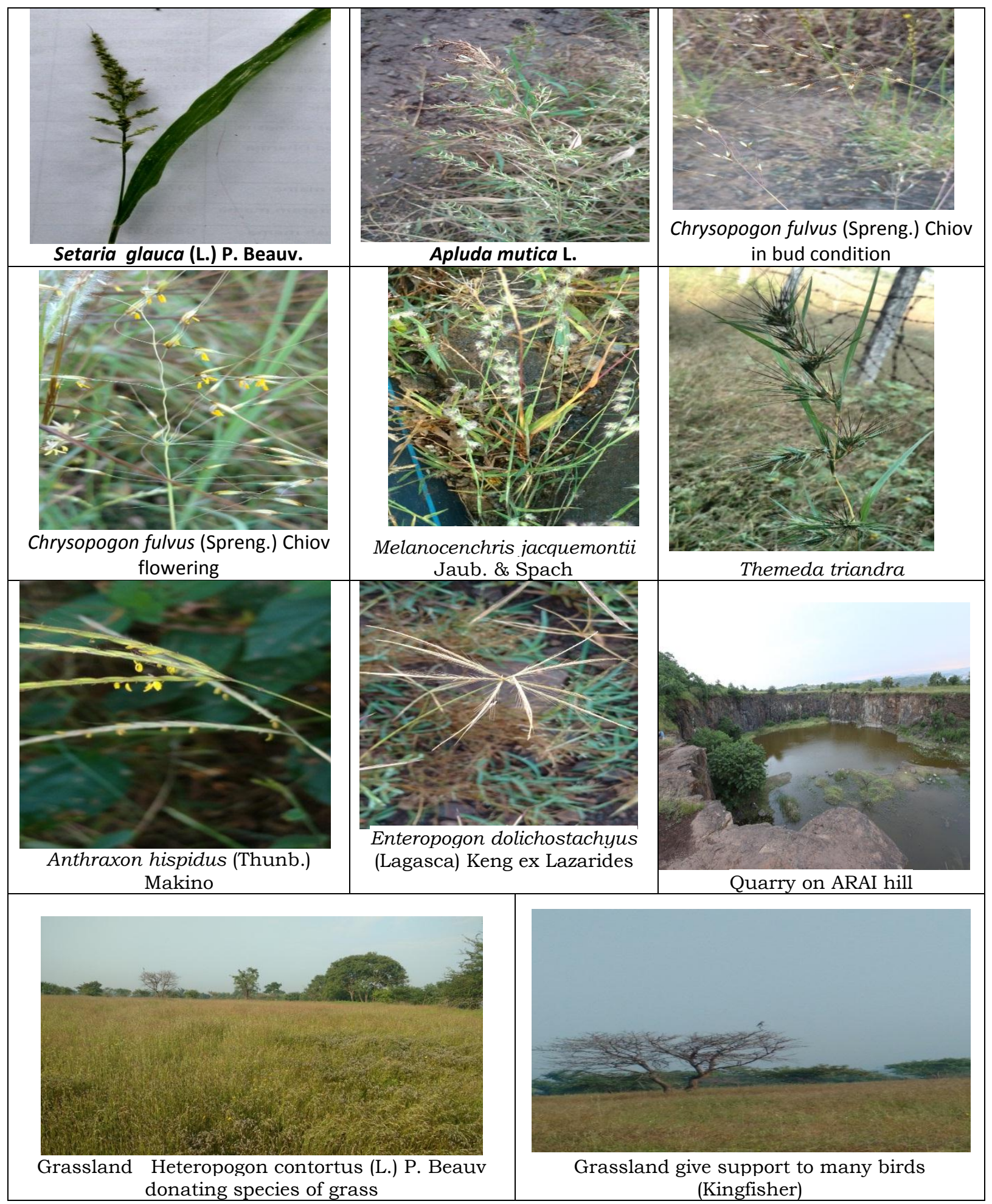

